\title{
SUMMABILITY AND FRACTIONAL LINEAR PARTIAL DIFFERENTIAL EQUATIONS
}

\author{
SEAWOMIR MICHALIK
}

\begin{abstract}
We consider the Cauchy problem for the Kowalevskaya type fractional linear partial differential equations in two complex variables with constant coefficients. We show that solution is analytically continued in some directions with exponential growth if and only if the similar properties satisfy the Cauchy data. Applying this result we study the summability of formal power series solution of a Cauchy problem for general non-Kowalevskian linear partial differential equations in normal form with constant coefficients. We obtain the necessary and sufficient conditions for the Borel summability in terms of analytic continuation with an appropriate growth condition of the Cauchy data. Moreover, we show the similar characterisation of Borel summability in the case of non-Kowalevskian fractional equations.
\end{abstract}

\section{IntRODUCTION}

In 1999 Lutz, Miyake and Schäfke [5] showed the first result in the theory of summability of formal power series solutions of partial differential equations. They proved that the formal solution to the Cauchy problem for the 1-dimensional homogeneous complex heat equation is 1-summable in a direction $d$ if and only if the Cauchy data $\varphi(z)$ can be analytically continued to infinity in some sectors in directions $d / 2$ and $d / 2+\pi$ and this continuation is of exponential growth of order at most 2 .

This characterisation was generalised to the equation $\partial_{t}^{p} u-\partial_{z}^{q} u=0$ (with $p<q$ ) by M. Miyake [8], to the quasi-homogeneous equations by K. Ichinobe [4] and to some linear partial differential equations by S. Michalik [7].

On the other hand, the sufficient condition for the Borel summability of formal solutions was found by Balser and Miyake [3] (for certain linear PDE with constant coefficients) and by W. Balser [2] (for general linear PDE with constant coefficients). In this last paper W. Balser also posed the conjecture that this sufficient condition for the Borel summability of formal power series solution is also necessary one.

In the paper we prove this conjecture in the case of equations in normal form. In this way, we also extend the results of M. Miyake [8], K. Ichinobe [4] and S. Michalik [7] to more general equations.

Namely, we consider the Cauchy problem for the non-Kowalevskian linear partial differential equation in two complex variables $t$ and $z$ with constant coefficients

(1) $\partial_{t}^{p} u(t, z)=\sum_{j=1}^{p} \partial_{t}^{p-j} P_{j q / p}\left(\partial_{z}\right) u(t, z), \quad \partial_{t}^{n} u(0, z)=\varphi_{n}(z), \quad n=0, \ldots, p-1$,

2000 Mathematics Subject Classification. 35E15, 35C10, 35C15, 26A33.

Key words and phrases. fractional linear PDE with constant coefficients, formal power series, Borel summability. 
where $p, q \in \mathbb{N}, p<q, P_{j q / p}(\xi)$ are polynomials of degree less than or equal to $j q / p$ $(j=1, \ldots, p), P_{q}(\xi)$ is a polynomial of degree $q$ and $\varphi_{n}(z)(n=0, \ldots, p-1)$ are analytic in some complex neighbourhood of the origin.

In a similar way to [7] we use the operator $B^{1,1+1 / k}$, which after appropriate change of variables is equal to the modified $k$-Borel transform $\mathcal{B}^{k}$. Applying $B^{1,1+1 / k}$ to the formal solution $\hat{u}(t, z)$ of the initial problem $(1)$ we obtain the associated function $v(t, z)$ satisfying the initial value problem for certain Kowalevskaya type fractional equation related to (1). It means that we can reduce the problem of summability to the study of this new equation. This concept is a generalisation of the idea given in [6], where the question about the summability of the formal solution to the heat equation is reduced to the investigation of the wave equation.

So, after this reduction we study the following fractional equation

$$
\left(\partial_{t}^{1 / p}\right)^{m} v(t, z)-\sum_{j=1}^{m}\left(\partial_{t}^{1 / p}\right)^{m-j} P_{j}\left(\partial_{z}^{1 / p}\right) v(t, z)=0,
$$

where $\operatorname{deg} P_{j}(\xi) \leq j, \operatorname{deg} P_{m}(\xi)=m$ and the initial data $\left(\partial_{t}^{1 / p}\right)^{n} v(0, z)=\varphi_{n}(z)$ $(n=0, \ldots, m-1)$ are $1 / p$-analytic in some complex neighbourhood of origin. We use the integral representation of the solution, which is based on the construction of Balser and Miyake [3]. Since this equation is in some sense symmetric with respect to both variables $t$ and $z$, we obtain the equivalence between the analytic continuation with appropriate growth condition of the Cauchy data $\varphi(z)$ and the similar properties of the solution $v(t, z)$ with respect to $t$. Precisely speaking, we have

Theorem (see Theorem 1). Let $\left\{\lambda_{1}, \ldots, \lambda_{l}\right\}$ be the set of the characteristic roots satisfying equation

$$
\lambda^{m}-\sum_{j=1}^{m} \lambda^{m-j} p_{j}=0 \quad \text { with } \quad p_{j}:=\lim _{\xi \rightarrow \infty} P_{j}(\xi) / \xi^{j} .
$$

Then the solution $v(t, z)$ of (2) is analytically continued in a direction $d$ with exponential growth of order $s>1$ as $t \rightarrow \infty$ if and only if the Cauchy data $\varphi_{n}(z)$ are analytically continued in directions $d+p \arg \lambda_{j}$ with exponential growth of order $s$ as $z \rightarrow \infty$.

The above result is a generalisation of Theorem 1 in [7] to fractional equations.

We can at last formulate the characterisation of Borel summability of formal power series solution of (1).

Theorem (see Theorem 2). The formal power series solution $\hat{u}(t, z)$ of the initial problem (1) is $p /(q-p)$-summable in a direction $d$ if and only if the Cauchy data $\varphi_{0}(z), \ldots, \varphi_{p-1}(z)$ are analytically continued in directions $\left(d+\arg \alpha_{j}+2 k \pi\right) p / q$ $(j=1, \ldots, l, k=0, \ldots, q-1)$ with exponential growth of order $q /(q-p)$, where $\left\{\alpha_{1}, \ldots, \alpha_{l}\right\}$ is the set of the roots of the characteristic equation

$$
\alpha^{p}-\sum_{j=1}^{p} \alpha^{p-j} \tilde{p}_{j}=0 \quad \text { with } \quad \tilde{p}_{j}:=\lim _{\xi \rightarrow \infty} P_{j q / p}(\xi) / \xi^{j q / p} .
$$

By Theorem 1, we can also show a similar characterisation of Borel summability of formal power series solutions of the Cauchy problem for the non-Kowalevskian fractional linear partial differential equation (see Propositions 8-10). 
In the paper we use the following notation. The complex (resp. real) disc in $\mathbb{C}^{n}$ (resp. $\mathbb{R}^{n}$ ) with a centre at origin and a radius $r>0$ is denoted by $D^{n}(r):=\{z \in$ $\left.\mathbb{C}^{n}:|z|<r\right\}$ (resp. $\left.B^{n}(r):=\left\{x \in \mathbb{R}^{n}:|x|<r\right\}\right)$. To simplify notation we write $D(r)$ for $n=1$. A sector in the universal covering space $\widetilde{\mathbb{C}}$ of $\mathbb{C} \backslash\{0\}$ is denoted by

$$
S(d, \varepsilon, R):=\left\{z \in \widetilde{\mathbb{C}}: z=r e^{i \theta}, d-\varepsilon / 2<\theta<d+\varepsilon / 2,0<r<R\right\}
$$

for $d \in \mathbb{R}, \varepsilon>0$ and $0<R \leq+\infty$. In the case of $R=+\infty$, we denote it briefly by $S(d, \varepsilon)$. A sector $S^{\prime}$ is called a proper subsector of $S(d, \varepsilon, R)$ if its closure in $\widetilde{\mathbb{C}}$ is contained in $S(d, \varepsilon, R)$.

By $\mathcal{O}(D)$ we denote the space of analytic functions on a domain $D \subseteq \mathbb{C}^{n}$. The Banach space of analytic functions on $D(r)$, continuous on its closure and equipped with the norm $\|\varphi\|_{r}:=\max _{|z| \leq r}|\varphi(z)|$ is denoted by $\mathbb{E}(r)$.

The space of formal power series

$$
\hat{u}(t, z)=\sum_{j=0}^{\infty} u_{j}(z) t^{j} \quad \text { with } \quad u_{j}(z) \in \mathbb{E}(r)
$$

is denoted by $\mathbb{E}(r)[[t]]$. Moreover, we set $\mathbb{E}[[t]]:=\bigcup_{r>0} \mathbb{E}(r)[[t]]$.

We denote by $P_{m}\left(\partial_{t}, \partial_{z}\right)$ the principal part of the differential operator $P\left(\partial_{t}, \partial_{z}\right)$ of order $m$. In other words, if $P\left(\partial_{t}, \partial_{z}\right)=\sum_{j+k<m} a_{j k} \partial_{t}^{j} \partial_{z}^{k}$ then $P_{m}\left(\partial_{t}, \partial_{z}\right)=$ $\sum_{j+k=m} a_{j k} \partial_{t}^{j} \partial_{z}^{k}$

\section{Gevrey formal power SERIES AND Borel Summability}

In this section we recall some fundamental facts about the Gevrey formal power series and the Borel summability. For more details we refer the reader to [1].

Definition 1. A function $u(t, z) \in \mathcal{O}(S(d, \varepsilon) \times D(r))$ is of exponential growth of order at most $s>0$ as $t \rightarrow \infty$ in $S(d, \varepsilon)$ if and only if for any $r_{1} \in(0, r)$ and any $\varepsilon_{1} \in(0, \varepsilon)$ there exist $A, B<\infty$ satisfying

$$
\max _{|z| \leq r_{1}}|u(t, z)|<A e^{B|t|^{s}} \quad \text { for every } \quad t \in S\left(d, \varepsilon_{1}\right)
$$

Analogously, a function $\varphi(z) \in \mathcal{O}(S(d, \varepsilon))$ is of exponential growth of order at most $s>0$ as $z \rightarrow \infty$ in $S(d, \varepsilon)$ if and only if for any $\varepsilon_{1} \in(0, \varepsilon)$ there exist $A, B<\infty$ such that

$$
|\varphi(z)|<A e^{B|z|^{s}} \quad \text { for every } \quad z \in S\left(d, \varepsilon_{1}\right) .
$$

Definition 2. Let $k>0$. A formal power series

$$
\hat{u}(t, z):=\sum_{j=0}^{\infty} u_{j}(z) t^{j} \quad \text { with } \quad u_{j}(z) \in \mathbb{E}(r)
$$

is $1 / k$-Gevrey formal power series in $t$ if its coefficients satisfy

$$
\max _{|z| \leq r}\left|u_{j}(z)\right| \leq A B^{j} \Gamma(1+j / k) \quad \text { for } \quad j=0,1, \ldots
$$

with some positive constants $A$ and $B$.

The set of $1 / k$-Gevrey formal power series in $t$ over $\mathbb{E}(r)$ is denoted by $\mathbb{E}(r)[[t]]_{1 / k}$. We also set $\mathbb{E}[[t]]_{1 / k}:=\bigcup_{r>0} \mathbb{E}(r)[[t]]_{1 / k}$. 
Definition 3. Let $k>0$ and $d \in \mathbb{R}$. A formal series $\hat{u}(t, z) \in \mathbb{E}[[t]]_{1 / k}$ defined by (3) is called $k$-summable in a direction $d$ if and only if its $k$-Borel transform

$$
\tilde{v}(t, z):=\sum_{j=0}^{\infty} u_{j}(z) \frac{t^{j}}{\Gamma(1+j / k)}
$$

is analytic in $S(d, \varepsilon) \times D(r)$ (for some $\varepsilon>0$ and $r>0$ ) and is of exponential growth of order at most $k$ as $t \rightarrow \infty$ in $S(d, \varepsilon)$. The $k$-sum of $\hat{u}(t, z)$ in the direction $d$ is represented by the Laplace transform of $\tilde{v}(t, z)$

$$
u^{\theta}(t, z):=\frac{1}{t^{k}} \int_{0}^{\infty(\theta)} e^{-(s / t)^{k}} \tilde{v}(s, z) d s^{k}
$$

where the integration is taken over any ray $e^{i \theta} \mathbb{R}_{+}:=\left\{r e^{i \theta}: r \geq 0\right\}$ with $\theta \in$ $(d-\varepsilon / 2, d+\varepsilon / 2)$.

For every $k>0$ and $d \in \mathbb{R}$, according to the general theory of moment summability (see Section 6.5 in [1]), a formal series (3) is $k$-summable in the direction $d$ if and only if the same holds for the series

$$
\sum_{j=0}^{\infty} u_{j}(z) \frac{j ! \Gamma(1+j / k)}{\Gamma(1+j(1+1 / k))} t^{j}
$$

Consequently, we obtain a characterisation of $k$-summability (analogous to Definition 3), if we replace the $k$-Borel transform by the modified $k$-Borel transform

$$
v(t, z):=\mathcal{B}^{k} \hat{u}(t, z):=\sum_{j=0}^{\infty} u_{j}(z) \frac{j ! t^{j}}{\Gamma(1+j(1+1 / k))}
$$

and the Laplace transform by the Ecalle acceleration operator

$$
u^{\theta}(t, z)=t^{-k /(1+k)} \int_{0}^{\infty(\theta)} v(s, z) C_{1+1 / k}\left((s / t)^{k /(1+k)}\right) d s^{k /(1+k)}
$$

with $\theta \in(d-\varepsilon, d+\varepsilon)$. Here integration is taken over the ray $e^{i \theta} \mathbb{R}_{+}$and $C_{1+1 / k}(\zeta)$ is defined by

$$
C_{1+1 / k}(\zeta):=\frac{1}{2 \pi i} \int_{\gamma} u^{-1 /(k+1)} e^{u-\zeta u^{k /(k+1)}} d u
$$

with a path of integration $\gamma$ as in the Hankel integral for the inverse Gamma function (from $\infty$ along $\arg u=-\pi$ to some $u_{0}<0$, then on the circle $|u|=\left|u_{0}\right|$ to $\arg u=\pi$, and back to $\infty$ along this ray).

Hence the $k$-summability can be characterised as follows

Proposition 1. Let $k>0$ and $d \in \mathbb{R}$. A formal series $\hat{u}(t, z)$ given by (3) is $k$-summable in a direction $d$ if and only if its modified $k$-Borel transform

$$
\mathcal{B}^{k} \hat{u}(t, z)=\sum_{j=0}^{\infty} u_{j}(z) \frac{j ! t^{j}}{\Gamma(1+j(1+1 / k))}
$$

satisfies conditions:

(1) $\mathcal{B}^{k} \hat{u}(t, z) \in \mathcal{O}\left(D\left(r_{1}\right) \times D\left(r_{2}\right)\right.$ ) (for some $r_{1}>0$ and $r_{2}>0$ ), i.e. $\hat{u}(t, z) \in$ $\mathbb{E}\left(r_{2}\right)[[t]]_{1 / k}$.

(2) $\mathcal{B}^{k} \hat{u}(t, z)$ is analytically continued to $S(d, \varepsilon) \times D(r)$ (for some $\varepsilon>0$ and $r>0)$.

(3) $\mathcal{B}^{k} \hat{u}(t, z)$ is of exponential growth of order at most $k$ as $t \rightarrow \infty$ in $S(d, \varepsilon)$. 


\section{3. $\alpha$-AnAlYtiC FUnCTIONS AND $\alpha$-DERIVATIVES}

In this section we introduce some kind of fractional derivatives $\partial_{z}^{\alpha}$ of the formal power series in $\mathbb{C}\left[\left[z^{\alpha}\right]\right]$. These operators are the natural generalisation of the derivative $\partial_{z}$ defined into the space $\mathbb{C}[[z]]$. Namely, we have

Definition 4. Let $\alpha>0$. The linear operator on the space of formal power series

$$
\partial_{z}^{\alpha}: \mathbb{C}\left[\left[z^{\alpha}\right]\right] \rightarrow \mathbb{C}\left[\left[z^{\alpha}\right]\right]
$$

given by the formula

$$
\partial_{z}^{\alpha}\left(\sum_{n=0}^{\infty} \frac{u_{n}}{\Gamma(1+\alpha n)} z^{\alpha n}\right)=\sum_{n=0}^{\infty} \frac{u_{n+1}}{\Gamma(1+\alpha n)} z^{\alpha n}
$$

is called an $\alpha$-derivative.

Definition 5. We say that a function $u(z)$ is $\alpha$-analytic on $D \subset \mathbb{C}$ (or, generally, on $\left.D \subset \mathbb{C}^{n}\right)$ if and only if the function $z \mapsto u\left(z^{1 / \alpha}\right)$ is analytic for every $z^{1 / \alpha} \in D$. The space of $\alpha$-analytic functions will be denoted by $\mathcal{O}_{\alpha}(D)$.

If the formal power series $\hat{u}(z) \in \mathbb{C}\left[\left[z^{\alpha}\right]\right]$ is convergent in some complex neighbourhood of origin, then its sum $u(z)$ is the $\alpha$-analytic function near the origin. For such functions we have well defined $\alpha$-derivative given by (4), which coincides with the Caputo fractional derivative.

We may also define the $\alpha$-Taylor series of $u(z) \in \mathcal{O}_{\alpha}(D)$ by the formula

$$
u(z)=\sum_{n=0}^{\infty} \frac{\left(\partial_{z}^{\alpha}\right)^{n} u(0)}{\Gamma(1+\alpha n)} z^{\alpha n}
$$

In the case of $\alpha$-analytic functions, the role of the exponential function $e^{z}$ is played by

$$
e_{\alpha}(z):=E_{\alpha}\left(z^{\alpha}\right)=\sum_{n=0}^{\infty} \frac{z^{\alpha n}}{\Gamma(1+\alpha n)},
$$

where $E_{\alpha}(z)$ denotes the Mittag-Leffler function. By the definition of $e_{\alpha}(z)$ and by the results on the Mittag-Leffler function (see [9]), we have

Proposition 2. The function $e_{\alpha}(z)$ satisfies the following properties:

(1) $e_{\alpha}(z) \in \mathcal{O}_{\alpha}(\mathbb{C})$ and there exists $C<\infty$ such that $\left|e_{\alpha}(z)\right| \leq C e^{|z|}$ for every $z \in \mathbb{C}$

(2) for every $a \in \mathbb{C}$ we have $\partial_{z}^{\alpha} e_{\alpha}(a z)=a^{\alpha} e_{\alpha}(a z)$ (in particular $\partial_{z}^{\alpha} e_{\alpha}(z)=$ $\left.e_{\alpha}(z)\right)$

(3) if $\alpha<2$ and $\arg z \in(\pi / 2,2 \pi / \alpha-\pi / 2)$ then $e_{\alpha}(z) \rightarrow 0$ as $z \rightarrow \infty$.

Let us assume that $\alpha \in \mathbb{Q}_{+}$. Since every $q / p$-analytic function is also $1 / p$ analytic, without loss of generality we may take $\alpha=1 / p$, where $p \in \mathbb{N}$. Observe that $1 / p$-analytic function is in fact an analytic function defined on the Riemann surface of $\sqrt[p]{z}$. Hence we may find the integral representation

Lemma 1. Let $\varphi(z) \in \mathcal{O}_{1 / p}(D(r))$. Then for every $|z|<\varepsilon<r$ and $k \in \mathbb{N}$ we have

$$
\left(\partial_{z}^{1 / p}\right)^{k} \varphi(z)=\frac{1}{2 p \pi i} \oint_{|w|=\varepsilon}^{p} \varphi(w) \int_{0}^{\infty(\theta)} \zeta^{k / p} e_{1 / p}(z \zeta) e^{-w \zeta} d \zeta d w
$$

for $\theta \in(\arg w-\pi / 2, \arg w+\pi / 2)$, where $\oint_{|w|=\varepsilon}^{p}$ denotes that we integrate $p$ times around the positively oriented circle of radius $\varepsilon$. 
Moreover, there exist $\varrho>0$ and $A, B<\infty$ satisfying

$$
\sup _{|z|<\varrho}\left|\left(\partial_{z}^{1 / p}\right)^{k} \varphi(z)\right| \leq A B^{k / p} \Gamma(1+k / p) \quad \text { for } \quad k=0,1, \ldots
$$

Proof. By the Cauchy integral formula on the Riemann surface of $\sqrt[p]{z}$ we have

$$
\varphi(0)=\frac{1}{2 p \pi i} \oint_{|w|=\varepsilon}^{p} \frac{\varphi(w)}{w} d w
$$

and more generally for every $n \in \mathbb{N}$

$$
\begin{aligned}
\left(\partial_{z}^{1 / p}\right)^{n} \varphi(0) & =\frac{\Gamma(1+n / p)}{2 p \pi i} \oint_{|w|=\varepsilon}^{p} \frac{\varphi(w)}{w^{n / p+1}} d w \\
& =\frac{1}{2 p \pi i} \oint_{|w|=\varepsilon}^{p} \varphi(w) \int_{0}^{\infty(\theta)} \zeta^{n / p} e^{-w \zeta} d \zeta d w
\end{aligned}
$$

with $\theta \in(\arg w-\pi / 2, \arg w+\pi / 2)$.

Hence, by (5)

$$
\begin{aligned}
\varphi(z) & =\sum_{n=0}^{\infty} \frac{\left(\partial_{z}^{1 / p}\right)^{n} \varphi(0)}{\Gamma(1+n / p)} z^{n / p}=\frac{1}{2 p \pi i} \oint_{|w|=\varepsilon}^{p} \varphi(w) \int_{0}^{\infty(\theta)} \sum_{n=0}^{\infty} \frac{(z \zeta)^{n / p}}{\Gamma(1+n / p)} e^{-w \zeta} d \zeta \\
& =\frac{1}{2 p \pi i} \oint_{|w|=\varepsilon}^{p} \varphi(w) \int_{0}^{\infty(\theta)} e_{1 / p}(z \zeta) e^{-w \zeta} d \zeta
\end{aligned}
$$

The first part of the proof is finished by the observation that $\left(\partial_{z}^{1 / p}\right)^{k} e_{1 / p}(z \zeta)=$ $\zeta^{k / p} e_{1 / p}(z \zeta)$.

To show the second part, note that by Proposition 2

$$
\begin{aligned}
\left|\int_{0}^{\infty(\theta)} \zeta^{k / p} e_{1 / p}(z \zeta) e^{-w \zeta} d \zeta\right| & \leq C \int_{0}^{\infty} \zeta^{k / p} e^{(|z|-|w|) \zeta} d \zeta \leq C \int_{0}^{\infty} \zeta^{k / p} e^{-\varepsilon \zeta / 2} d \zeta \\
& =C \frac{\Gamma(1+k / p)}{(\varepsilon / 2)^{k / p+1}}
\end{aligned}
$$

for $|z|<\varepsilon / 2,|w|=\varepsilon$ and $\theta=\arg w$. It means that for $\varrho:=\varepsilon / 2$ we have

$$
\sup _{|z|<\varrho}\left|\left(\partial_{z}^{1 / p}\right)^{k} \varphi(z)\right| \leq \frac{C}{2 p \pi} \oint_{|w|=\varepsilon}^{p}|\varphi(w)| \frac{\Gamma(1+k / p)}{(\varepsilon / 2)^{k / p+1}} d w \leq A B^{k / p} \Gamma(1+k / p)
$$

with some positive constants $A, B<\infty$.

\section{Operators $B^{\alpha, \beta}$}

In this section we introduce the operators $B^{\alpha, \beta}$ with $\alpha, \beta>0$, which are related to the modified $k$-Borel operators $\mathcal{B}^{k}$. Using the operators $B^{\alpha, \beta}$ we can reduce the question about summability to the study of the solution of the appropriate Kowalevskaya type equation.

Definition 6. Let $\alpha, \beta>0$. We define a linear operator on the space of formal power series

by the formula

$$
B^{\alpha, \beta}: \mathbb{E}\left[\left[t^{\alpha}\right]\right] \rightarrow \mathbb{E}\left[\left[t^{\beta}\right]\right]
$$

$$
B^{\alpha, \beta}(\hat{u}(t, z))=B^{\alpha, \beta}\left(\sum_{n=0}^{\infty} \frac{u_{n}(z)}{\Gamma(1+\alpha n)} t^{\alpha n}\right):=\sum_{n=0}^{\infty} \frac{u_{n}(z)}{\Gamma(1+\beta n)} t^{\beta n} .
$$


Observe that for any formal series $\hat{u}(t, z) \in \mathbb{E}[[t]]$ and $k>0$ holds

$$
\mathcal{B}^{k} \hat{u}(t, z)=B^{1, s} \hat{u}\left(t^{1 / s}, z\right) \quad \text { with } \quad s=1+1 / k .
$$

In particular, taking $s=q / p$ and using the operator $B^{1, q / p}: \mathbb{E}[[t]] \rightarrow \mathbb{E}\left[\left[t^{q / p}\right]\right]$ given by

$$
B^{1, q / p}\left(\sum_{j=0}^{\infty} \frac{u_{n}(z)}{n !} t^{n}\right)=\sum_{n=0}^{\infty} \frac{u_{n}(z)}{\Gamma(1+n q / p)} t^{n q / p},
$$

one can reformulate Proposition 1 as follows

Proposition 3. Let $p, q \in \mathbb{N}, p<q$. Then the formal series $\hat{u}(t, z) \in \mathbb{E}[[t]]$ is $p /(q-p)$-summable in a direction $d$ if and only if the function $v(t, z):=B^{1, q / p} \hat{u}(t, z)$ satisfies the following properties:

(1) $z \mapsto v(t, z)$ is analytic in some complex neighbourhood of origin,

(2) $t \rightarrow v(t, z)$ is $q / p$-analytic in some complex neighbourhood of origin,

(3) $t \rightarrow v(t, z)$ is $q / p$-analytically continued to infinity in directions $(d+2 k \pi) p / q$ $(k=0, \ldots, q-1)$ with exponential growth of order $q /(q-p)$.

The following properties of the operators $B^{\alpha, \beta}$ play crucial role in our study of summability.

Proposition 4. Let $\alpha, \beta>0$ and $\hat{u}(t, z) \in \mathbb{E}\left[\left[t^{\alpha}\right]\right]$. Then operators $B^{\alpha, \beta}$ and derivatives satisfy the following commutation formulas:

(1) $B^{\alpha, \beta} \partial_{t}^{\alpha} \hat{u}(t, z)=\partial_{t}^{\beta} B^{\alpha, \beta} \hat{u}(t, z)$;

(2) $B^{\alpha, \beta} \partial_{z} \hat{u}(t, z)=\partial_{z} B^{\alpha, \beta} \hat{u}(t, z)$;

(3) $B^{\alpha, \beta} P\left(\partial_{t}^{\alpha}, \partial_{z}\right) \hat{u}(t, z)=P\left(\partial_{t}^{\beta}, \partial_{z}\right) B^{\alpha, \beta} \hat{u}(t, z)$ for any polynomial $P(\tau, \zeta):=$ $\sum_{j=1}^{m} \sum_{l=1}^{n} a_{j l} \tau^{j} \zeta^{l}$ with constant coefficients $a_{j l} \in \mathbb{C}$.

Proof. From (6) we see that

$$
\begin{aligned}
& B^{\alpha, \beta}\left(\partial_{t}^{\alpha} \hat{u}(t, z)\right)=B^{\alpha, \beta}\left(\sum_{n=0}^{\infty} \frac{u_{n+1}(z)}{\Gamma(1+\alpha n)} t^{\alpha n}\right)=\sum_{n=0}^{\infty} \frac{u_{n+1}(z)}{\Gamma(1+\beta n)} t^{\beta n} \\
& =\partial_{t}^{\beta} B^{\alpha, \beta}(\hat{u}(t, z))
\end{aligned}
$$

and

$$
B^{\alpha, \beta}\left(\partial_{z} \hat{u}(t, z)\right)=\sum_{n=0}^{\infty} \frac{\partial_{z} u_{n}(z)}{\Gamma(1+\beta n)} t^{\beta n}=\partial_{z} B^{\alpha, \beta}(\hat{u}(t, z)) .
$$

Consequently

$$
\begin{aligned}
& B^{\alpha, \beta}\left(P\left(\partial_{t}^{\alpha}, \partial_{z}\right) \hat{u}(t, z)\right)=B^{\alpha, \beta}\left(\sum_{j=1}^{m} \sum_{l=1}^{n} a_{j l}\left(\partial_{t}^{\alpha}\right)^{j} \partial_{z}^{l} \hat{u}(t, z)\right) \\
& =\sum_{j=1}^{m} \sum_{l=1}^{n} a_{j l} B^{\alpha, \beta}\left(\left(\partial_{t}^{\alpha}\right)^{j} \partial_{z}^{l} \hat{u}(t, z)\right)=\sum_{j=1}^{m} \sum_{l=1}^{n} a_{j l}\left(\partial_{t}^{\beta}\right)^{j} \partial_{z}^{l} B^{\alpha, \beta}(\hat{u}(t, z)) \\
& =P\left(\partial_{t}^{\beta}, \partial_{z}\right) B^{\alpha, \beta}(\hat{u}(t, z)) .
\end{aligned}
$$

By Proposition 4 we have 
Proposition 5. A formal series $\hat{u}(t, z)$ is a solution of the Cauchy problem (1) for the non-Kowalevskian linear partial differential equation with constant coefficients if and only if the formal series $\hat{v}(t, z):=B^{1, q / p}(\hat{u}(t, z))$ satisfies the following fractional equation

$$
\begin{aligned}
& \left(\partial_{t}^{1 / p}\right)^{q p} v(t, z)=\sum_{j=1}^{p}\left(\partial_{t}^{1 / p}\right)^{q(p-j)} P_{j q / p}\left(\left(\partial_{z}^{1 / p}\right)^{p}\right) v(t, z), \\
& \left(\partial_{t}^{1 / p}\right)^{j} v(0, z)=\varphi_{n}(z) \text { for } j=n q, \\
& \left(\partial_{t}^{1 / p}\right)^{j} v(0, z)=0 \text { for } j \neq n q, j<q p, n=0, \ldots, p-1 .
\end{aligned}
$$

\section{The SOLUTION OF FRACTIONAL EQUATION}

In this section we consider the initial value problem to fractional equation

$$
P\left(\partial_{t}^{1 / p}, \partial_{z}^{1 / p}\right) v(t, z)=0
$$

(9) $\left(\partial_{t}^{1 / p}\right)^{n} v(0, z)=\varphi_{n}(z) \in \mathcal{O}_{1 / p}(D(r)) \quad$ for some $r>0 \quad(n=0, \ldots, m-1)$,

where

$$
P\left(\partial_{t}^{1 / p}, \partial_{z}^{1 / p}\right):=\left(\partial_{t}^{1 / p}\right)^{m}-\sum_{j=1}^{m}\left(\partial_{t}^{1 / p}\right)^{m-j} P_{j}\left(\partial_{z}^{1 / p}\right)
$$

with $\operatorname{deg} P_{j}(\xi) \leq j$ and $\operatorname{deg} P_{m}(\xi)=m$. We would like to find the relation between the Cauchy data and solution. For this purpose we will use an integral representation of the solution of (8) with the initial data $\varphi_{n}(z)$ given by the recurrence relations

(10) $\varphi_{0}(z):=\varphi(z), \quad \varphi_{n}(z):=\sum_{j=1}^{n} P_{j}\left(\partial_{z}^{1 / p}\right) \varphi_{n-j}(z)$ for $n=1, \ldots, m-1$,

where $\varphi(z) \in \mathcal{O}_{1 / p}(D(r))$. The construction of this integral representation is based upon the results of Balser and Miyake [3]. This method is also similar to that used in our previous paper [7], where the case of differential equations $(p=1)$ was considered.

We start with the following version of the Cauchy-Kowalevskaya theorem

Lemma 2. The formal power series solution

$$
\hat{v}(t, z)=\sum_{n=0}^{\infty} v_{n}(z) \frac{t^{n / p}}{\Gamma(1+n / p)}
$$

of (8) with the initial data (9) is convergent in some neighbourhood of origin.

Proof. By the principle of superposition of solutions of linear equations, we may assume that the initial data satisfy $(10)$. Note that coefficients $v_{n}(z)$ satisfy the recurrence relation

$$
v_{n}(z)=\sum_{j=1}^{m} P_{j}\left(\partial_{z}^{1 / p}\right) v_{n-j}(z) \quad \text { for } \quad n=1,2, \ldots
$$

with $v_{-m+1}(z)=\ldots=v_{-1}(z)=0$ and $v_{0}(z)=\varphi(z)$. It means that

$$
v_{n}(z)=q_{n}\left(\partial_{z}^{1 / p}\right) \varphi(z) \text { for } n=1,2, \ldots
$$


where $q_{n}(\xi)$ is a solution of the difference equation

$$
q_{n}(\xi)=\sum_{j=1}^{m} P_{j}(\xi) q_{n-j}(\xi)
$$

with the initial conditions

$$
q_{0}(\xi)=1 \quad \text { and } \quad q_{-1}(\xi)=\ldots=q_{-m+1}(\xi)=0 .
$$

Observe that $q_{n}(\xi)$ is a polynomial of degree less than or equal to $n$, so $q_{n}(\xi)=$ $\sum_{k=0}^{n} q_{n k} \xi^{k}$ with some constant coefficients $q_{n k}$.

Put $Q_{n}(\xi):=\sum_{k=0}^{n}\left|q_{n k}\right| \xi^{k}$. Since $Q_{n}(\xi)$ is a polynomial of degree $n$, there exists $K>0$ such that

$$
\left|Q_{n}(\xi)\right| \leq\left(K^{1 / p} \xi\right)^{n} \quad \text { for every } \quad n \in \mathbb{N} \text { and } \xi>1 .
$$

By Lemma 1 , there exist $\varrho>0$ and $A, B<\infty$ satisfying

$$
\begin{aligned}
\sup _{|z|<\varrho}\left|\hat{v}_{n}(z)\right| & \leq \sum_{k=0}^{n}\left|q_{n k}\right| \sup _{|z|<\varrho}\left|\left(\partial_{z}^{1 / p}\right)^{k} \varphi(z)\right| \leq \sum_{k=0}^{n}\left|q_{n k}\right| A B^{k / p} \Gamma(1+k / p) \\
& \leq A \Gamma(1+n / p) Q_{n}\left(B^{1 / p}\right) \leq A(K B)^{n / p} \Gamma(1+n / p) .
\end{aligned}
$$

Therefore, the formal series (11) is convergent for $|t|<(K B)^{-1}$ and $|z|<\varrho$.

Lemma 3. Let $s>1, d \in \mathbb{R}$ and $(t, z) \in D^{2}(r)$ (for some $r>0$ ). Suppose that $v(t, z)$ is a solution of (8) with the initial data (10) and that $\left\{\lambda_{1}, \ldots, \lambda_{l}\right\}$ is the set of the roots of the characteristic equation

$$
P_{m}(\lambda, 1)=\lambda^{m}-\sum_{j=1}^{m} \lambda^{m-j} p_{j}=0 \quad \text { with } \quad p_{j}:=\lim _{\xi \rightarrow \infty} P_{j}(\xi) / \xi^{j} .
$$

Then $v(t, z)=\sum_{j=1}^{l} v_{j}(t, z)$, where $v_{j}(t, z) \in \mathcal{O}_{1 / p}\left(D^{2}(r)\right)$ satisfy the following property: if $\varphi(z) \in \mathcal{O}_{1 / p}\left(S\left(d+\arg \lambda_{j}, \tilde{\delta}\right)\right)$ (for some $\tilde{\delta}>0$ ) with an exponential growth of order $s$ in infinity then $v_{j}(t, z) \in \mathcal{O}_{1 / p}(S(d, \delta) \times D(r)$ ) (for some $\delta>0$ and $r>0$ ) with the same exponential growth as $t \rightarrow \infty$.

Proof. By Lemma 2, for sufficiently small $t$ and $z$, the power series (11) is convergent. Hence, using (12) and applying Lemma 1, one can choose $\varepsilon>0$ such that

(16) $v(t, z)=\sum_{n=0}^{\infty} \frac{t^{n / p}}{\Gamma(1+n / p)} \sum_{k=0}^{n} q_{n k}\left(\partial_{z}^{1 / p}\right)^{k} \varphi(z)=\frac{1}{2 p \pi i} \oint_{|w|=\varepsilon}^{p} \varphi(w) k(t, w, z) d w$,

where the kernel function is defined by

$$
\begin{aligned}
k(t, w, z) & :=\sum_{n=0}^{\infty} \frac{t^{n / p}}{\Gamma(1+n / p)} \sum_{k=0}^{n} q_{n k} \int_{0}^{\infty(\theta)} \zeta^{k / p} e_{1 / p}(z \zeta) e^{-w \zeta} d \zeta \\
& =\int_{0}^{\infty(\theta)} q(t, \zeta) e_{1 / p}(z \zeta) e^{-w \zeta} d \zeta
\end{aligned}
$$

with $\theta=-\arg w$ and

$$
q(t, \zeta):=\sum_{n=0}^{\infty} \frac{t^{n / p}}{\Gamma(1+n / p)} \sum_{k=0}^{n} q_{n k} \zeta^{k / p}=\sum_{n=0}^{\infty} \frac{t^{n / p}}{\Gamma(1+n / p)} q_{n}\left(\zeta^{1 / p}\right)
$$


By (15) we may estimate

$$
|q(t, \zeta)| \leq \sum_{n=0}^{\infty} \frac{|t|^{n / p}(K|\zeta|)^{n / p}}{\Gamma(1+n / p)}=e_{1 / p}(K|t||\zeta|)
$$

Hence, by Proposition 2, $q(t, \zeta) \in \mathcal{O}_{1 / p}\left(\mathbb{C}^{2}\right)$ is of exponential order 1. Moreover, for $|z|<|w|$ and $\theta=-\arg w$ we have

$$
\left|\int_{0}^{\infty(\theta)} q(t, \zeta) e_{1 / p}(z \zeta) e^{-w \zeta} d \zeta\right|<\infty \quad \text { for } \quad K|t|+|z|<|w| .
$$

It means that under above conditions on $w$ and $z$, the function $t \rightarrow k(t, w, z)$ is $1 / p$-analytic on the set $\{t \in \mathbb{C}: K|t|+|z|<|w|\}$.

On the other hand, using the characteristic equation

$$
P(\lambda, \xi)=\lambda^{m}-\sum_{j=1}^{m} P_{j}(\xi) \lambda^{m-j}=0
$$

one can find the solution of (13). We may assume that for sufficiently large $|\xi|$, say $|\xi|>\left|\zeta_{0}^{1 / p}\right|$, the characteristic equation (17) has exactly $l$ distinct holomorphic solutions $\lambda_{1}(\xi), \ldots, \lambda_{l}(\xi)$ of multiplicity $m_{1}, \ldots, m_{l}\left(\sum_{j=1}^{l} m_{j}=m\right)$. Since $P_{m}(\xi) \neq$ 0 , we conclude that $\lambda_{j}(\xi) \not \equiv 0$. Moreover, $\operatorname{deg} P_{j}(\xi) \leq j$ and $\operatorname{deg} P_{m}(\xi)=m$, which gives

$$
\lim _{\xi \rightarrow \infty} \lambda_{j}(\xi) / \xi=\lambda_{j} \in \mathbb{C} \backslash\{0\},
$$

where $\lambda_{j}$ are the roots of the characteristic equation

$$
P_{m}(\lambda, 1)=\lambda^{m}-\sum_{j=1}^{m} p_{j} \lambda^{m-j}=0 \quad \text { with } \quad p_{j}:=\lim _{\xi \rightarrow \infty} P_{j}(\xi) / \xi^{j} .
$$

Note that $\lambda_{j}$ are not necessarily the distinct roots of (19). From (18) we can also assume that for $|\xi|>\left|\zeta_{0}^{1 / p}\right|$ the functions $\lambda_{j}(\xi)$ are invertible, where the inverse functions $\lambda_{j}^{-1}(\tau)$ are the roots of the characteristic equation $P\left(\tau, \lambda^{-1}\right)=0$.

Using the roots of the characteristic equation (17) one can find $m$ linear independent solutions of the difference equation (13)

$$
\lambda_{j}^{n}(\xi), n \lambda_{j}^{n}(\xi), \ldots, n^{m_{j}-1} \lambda_{j}^{n}(\xi) \quad \text { for } \quad j=1, \ldots, l .
$$

Hence for $|\zeta|>\left|\zeta_{0}\right|$ and $\zeta^{1 / p}=\xi$, the solution of (13) is given by

$$
q_{n}\left(\zeta^{1 / p}\right)=\sum_{j=1}^{l} \sum_{k=0}^{m_{j}-1} c_{j k}\left(\zeta^{1 / p}\right) n^{k} \lambda_{j}^{n}\left(\zeta^{1 / p}\right) .
$$

We can calculate the coefficients $c_{j k}\left(\zeta^{1 / p}\right)$ using the initial conditions (14) and solving the system of linear equations. Observe that for sufficiently large $|\zeta|$, say $|\zeta|>\left|\zeta_{0}\right|$, the coefficients $c_{j k}\left(\zeta^{1 / p}\right)$ are holomorphic with polynomial growth as $|\zeta| \rightarrow \infty$.

Moreover, since

$$
n t^{n / p}=\left(\left(\partial_{t}^{1 / p}\right)^{p} t-1\right) p t^{n / p}=: r\left(t, \partial_{t}^{1 / p}\right) t^{n / p}
$$


we have

$$
q(t, \zeta)=\sum_{j=1}^{l} \sum_{k=0}^{m_{j}-1} c_{j k}\left(\zeta^{1 / p}\right) r^{k}\left(t, \partial_{t}^{1 / p}\right) e_{1 / p}\left(t \lambda_{j}^{p}\left(\zeta^{1 / p}\right)\right) \quad \text { for } \quad|\zeta|>\left|\zeta_{0}\right| .
$$

Hence

$$
\begin{aligned}
& k(t, w, z)=\int_{0}^{\zeta_{0}} q(t, \zeta) e_{1 / p}(z \zeta) e^{-w \zeta} d \zeta \\
& +\sum_{j=1}^{l} \sum_{k=0}^{m_{j}-1} \int_{\zeta_{0}}^{\infty(\theta)} c_{j k}\left(\zeta^{1 / p}\right)\left(p t\left(\partial_{t}^{1 / p}\right)^{p}\right)^{k} e_{1 / p}\left(t \lambda_{j}^{p}\left(\zeta^{1 / p}\right)\right) e_{1 / p}(z \zeta) e^{-w \zeta} d \zeta
\end{aligned}
$$

Fix $w, z \in \mathbb{C}$ such that $|z|$ is small relative to $|w|=\varepsilon$. It means that $|w|-|z| \approx|w|$ and $\arg (w-z) \approx \arg w$. To show analytic continuation of $k(t, w, z)$ with respect to $t$, we will consider the function

$$
a_{j}(t, z, w)=\int_{\zeta_{0}}^{\infty(\theta)} e_{1 / p}\left(t \lambda_{j}^{p}\left(\zeta^{1 / p}\right)\right) e_{1 / p}(z \zeta) e^{-w \zeta} d \zeta
$$

To estimate $a_{j}(t, w, z)$ for $j=1, \ldots, l$, observe that by (15), (18) and (20) we have $\left|\lambda_{j}\right|<K^{1 / p}$ and consequently the function $t \mapsto a_{j}(t, w, z)$ is analytic on $\{t \in \mathbb{C}:|t|<|w| / K\}$ for $j=1, \ldots, l$. Moreover, by (18), for sufficiently large $\zeta$ we have $\arg \lambda_{j}^{p}\left(\zeta^{1 / p}\right) \approx \arg \zeta+p \arg \lambda_{j}$. Now we want to replace a direction $\theta$ in $(21)$ by $\theta_{j}$ satisfying:

(1) $\arg t+p \arg \lambda_{j}+\theta_{j} \in\left(\frac{\pi}{2}, 2 p \pi-\frac{\pi}{2}\right)$ modulo $2 p \pi$ (in this case by Proposition 2 we have $e_{1 / p}\left(t \lambda_{j}^{p}\left(\zeta^{1 / p}\right)\right) \rightarrow 0$ as $\left.\zeta \rightarrow \infty, \arg \zeta=\theta_{j}\right)$,

(2) $\arg w+\theta_{j} \in\left(-\frac{\pi}{2}, \frac{\pi}{2}\right)$ modulo $2 \pi$ (in this case there exists $\epsilon>0$ such that $\left|e_{1 / p}(z \zeta) e^{-w \zeta}\right| \leq e^{-\epsilon|\zeta|}$ as $\left.\zeta \rightarrow \infty, \arg \zeta=\theta_{j}\right)$.

Observe that these requirements may be together satisfied under the condition that $\arg t+p \arg \lambda_{j} \neq \arg z$ modulo $2 p \pi$. Therefore the function $t \mapsto a_{j}(t, w, z)$ is $1 / p$ analytically continued to $\left\{t \in \widetilde{\mathbb{C}}: \arg t \neq \arg w-p \arg \lambda_{j}\right.$ modulo $\left.2 p \pi\right\}$ with exponential growth of order 1 . It means that also the function

$$
t \rightarrow k_{j}(t, w, z):=\sum_{k=0}^{m_{j}-1} \int_{\zeta_{0}}^{\infty\left(\theta_{j}\right)} c_{j k}\left(\zeta^{1 / p}\right) r^{k}\left(t, \partial_{t}^{1 / p}\right) e_{1 / p}\left(t \lambda_{j}^{p}\left(\zeta^{1 / p}\right)\right) e_{1 / p}(z \zeta) e^{-w \zeta} d \zeta
$$

is $1 / p$-analytically continued to the same sector with exponential growth of order 1 .

By the Cauchy theorem

$$
\frac{1}{2 p \pi i} \oint_{|w|=\varepsilon}^{p} \varphi(w) \int_{0}^{\zeta_{0}} q(t, \zeta) e_{1 / p}(z \zeta) e^{-w \zeta} d \zeta d w=0
$$

Hence, by (16), for $t$ and $z$ close to origin, $|z|<\varepsilon$ and sufficiently small $\varepsilon>0$, the solution of (8) with the initial conditions (10) satisfies

$$
\begin{aligned}
v(t, z) & =\frac{1}{2 p \pi i} \oint_{|w|=\varepsilon}^{p} \varphi(w) k(t, w, z) d w=\sum_{j=1}^{l} \frac{1}{2 p \pi i} \oint_{|w|=\varepsilon}^{p} \varphi(w) k_{j}(t, w, z) d w \\
& =\sum_{j=1}^{l} v_{j}(t, z),
\end{aligned}
$$


where

$$
\begin{aligned}
v_{j}(t, z):= & \frac{1}{2 p \pi i} \oint_{|w|=\varepsilon}^{p} \varphi(w) \times \\
(22) \quad & \times \sum_{k=0}^{m_{j}-1} \int_{\zeta_{0}}^{\infty\left(\theta_{j}\right)} c_{j k}\left(\zeta^{1 / p}\right) r^{k}\left(t, \partial_{t}^{1 / p}\right) e_{1 / p}\left(t \lambda_{j}^{p}\left(\zeta^{1 / p}\right)\right) e_{1 / p}(z \zeta) e^{-w \zeta} d \zeta d w .
\end{aligned}
$$

To estimate $v_{j}(t, z)$, fix $z$ such close to the origin, that $\arg (w-z) \approx \arg w$ along a circle $|w|=\varepsilon$. Repeating the proof of Theorem 3.1 in [3], we split this circle into $2 \operatorname{arcs} \gamma$ and $\tilde{\gamma}$, where $\gamma$ extends between points of argument $d+p \arg \lambda_{j}-\tilde{\delta} / 3$ and $d+p \arg \lambda_{j}+\tilde{\delta} / 3$. Finally, since $\varphi(z) \in \mathcal{O}_{1 / p}\left(S\left(d+p \arg \lambda_{j}, \tilde{\delta}\right)\right)$, we may deform $\gamma$ into a path $\gamma_{R}$ along the ray $\arg w=d+\arg \lambda_{j}-\tilde{\delta} / 3$ to a point with modulus $R$ (which can be chosen arbitrarily large), then along the circle $|w|=R$ to the ray $\arg w=d+\arg \lambda_{j}+\tilde{\delta} / 3$ and back along this ray to the original circle. So, we have

$$
\begin{aligned}
v_{j}(t, z) & =\frac{1}{2 p \pi i} \oint_{|w|=\varepsilon}^{p} \varphi(w) k_{j}(t, w, z) d w \\
& =\frac{1}{2 p \pi i} \oint_{\gamma_{R}} \varphi(w) k_{j}(t, w, z) d w+\frac{1}{2 p \pi i} \oint_{\tilde{\gamma}} \varphi(w) k_{j}(t, w, z) d w .
\end{aligned}
$$

Note that $R$ may be chosen arbitrarily large and the function $t \mapsto k_{j}(t, w, z)$ is analytic on $|t|<|w| / K$, where $|z|$ is small relative to $|w|$. Hence, one can find $\delta>0$ such that the first integral on the right-hand side is $1 / p$-analytically continued to $S(d, \delta) \times D(r)$. Estimating this integral we see that it is of exponential growth of order at most $s$ as $t \rightarrow \infty$.

Moreover, since the function $t \mapsto k_{j}(t, w, z)$ is analytically continued into the region $\left\{t \in \widetilde{\mathbb{C}}: \arg t \neq \arg w-p \arg \lambda_{j}\right.$ modulo $\left.2 p \pi\right\}$, we see that the second integral on the right-hand side is also $1 / p$-analytically continued to $S(d, \delta) \times D(r)$ with appropriate estimation as $t \rightarrow \infty$. It means that $v_{j}(t, z)$ is $1 / p$-analytically continued to $S(d, \delta) \times D(r)$ with exponential growth of order $s$ as $t \rightarrow \infty$

Now, in a similar way to [7], we introduce some kind of the pseudodifferential operators connected with $1 / p$-derivative. To this end, let $\lambda\left(\zeta^{1 / p}\right)$ be a non-vanishing $1 / p$-analytic function for $|\zeta| \geq\left|\zeta_{0}\right|$ with a polynomial growth in infinity. Moreover, we assume that $\varphi(w) \in \mathcal{O}_{1 / p}(D(\tilde{r}))$ (with some $\left.\tilde{r}>0\right), f(\zeta, t, w) \in \mathcal{O}_{1 / p}(\mathbb{C} \times D(r) \times$ $D(\tilde{r})$ ) (with some $r>0$ and $\tilde{r}>0$ ) and a function

$$
v(t, z)=\frac{1}{2 p \pi i} \oint_{|w|=\varepsilon}^{p} \varphi(w) \int_{\zeta_{0}}^{\infty(\theta)} f(\zeta, t, w) e_{1 / p}(z \zeta) d \zeta d w
$$

is well-defined and $1 / p$-analytic in some complex neighbourhood of origin. Then we can define a pseudodifferential operator $\lambda\left(\partial_{z}^{1 / p}\right)$ acting on $v(t, z)$ as follows

$$
\lambda\left(\partial_{z}^{1 / p}\right) v(t, z):=\frac{1}{2 p \pi i} \oint_{|w|=\varepsilon}^{p} \varphi(w) \int_{\zeta_{0}}^{\infty(\theta)} \lambda\left(\zeta^{1 / p}\right) f(\zeta, t, w) e_{1 / p}(z \zeta) d \zeta d w .
$$

Remark 1. Let $s>1$. Observe that $v(t, z) \in \mathcal{O}_{1 / p}(D(r) \times S(d, \delta))$ with exponential growth of order $s$ if and only if $\lambda\left(\partial_{z}^{1 / p}\right) v(t, z)$ has the same properties.

Remark 2. Using the pseudodifferential operators we can write

$$
P\left(\partial_{t}^{1 / p}, \partial_{z}^{1 / p}\right) v(t, z)=\left(\partial_{t}^{1 / p}-\lambda_{1}\left(\partial_{z}^{1 / p}\right)\right)^{m_{1}} \ldots\left(\partial_{t}^{1 / p}-\lambda_{l}\left(\partial_{z}^{1 / p}\right)\right)^{m_{l}} v(t, z)=0 .
$$


We show

Lemma 4. The functions $v_{j}(t, z)$ given by (22) are the solutions of pseudodifferential equations

$$
\left(\partial_{t}^{1 / p}-\lambda_{j}\left(\partial_{z}^{1 / p}\right)\right)^{m_{j}} v_{j}(t, z)=0 \quad \text { for } \quad j=1, \ldots, l .
$$

Proof. By (22) and (23) we have

$$
\begin{aligned}
& \left(\partial_{t}^{1 / p}-\lambda_{j}\left(\partial_{z}^{1 / p}\right)\right)^{m_{j}} v_{j}(t, z) \\
& =\frac{1}{2 p \pi i} \oint_{|w|=\varepsilon} \varphi(w) \int_{\zeta_{0}}^{\infty\left(\theta_{j}\right)} \sum_{k=0}^{m_{j}-1} c_{j k}\left(\zeta^{1 / p}\right) b_{j k}(t, \zeta) e_{1 / p}(z \zeta) e^{-w \zeta} d \zeta d w,
\end{aligned}
$$

where

$$
b_{j k}(t, \zeta)=\left(\partial_{t}^{1 / p}-\lambda_{j}\left(\zeta^{1 / p}\right)\right)^{m_{j}} \sum_{n=0}^{\infty} \frac{n^{k} \lambda_{j}^{n}\left(\zeta^{1 / p}\right) t^{n / p}}{\Gamma(1+n / p)} .
$$

Next, for any fixed $k \in \mathbb{N}_{0}$ we define the family of polynomials

$$
P_{k, 0}(n):=n^{k}, \quad P_{k, j}(n):=P_{k, j-1}(n+1)-P_{k, j-1}(n) \quad \text { for } j \in \mathbb{N} .
$$

Observe that $\operatorname{deg} P_{k, j}(\xi)=\max \{k-j,-1\}$, where $\operatorname{deg} P_{k, j}(\xi)=-1$ if and only if $P_{k, j}(\xi) \equiv 0$.

Using these polynomials we can write

$$
\begin{aligned}
b_{j k}(t, \zeta) & =\left(\partial_{t}^{1 / p}-\lambda_{j}\left(\zeta^{1 / p}\right)\right)^{m_{j}} \sum_{n=0}^{\infty} \frac{P_{k, 0}(n) \lambda_{j}^{n}\left(\zeta^{1 / p}\right) t^{n / p}}{\Gamma(1+n / p)}=\ldots \\
& =\left(\partial_{t}^{1 / p}-\lambda_{j}\left(\zeta^{1 / p}\right)\right)^{m_{j}-k-1} \sum_{n=0}^{\infty} \frac{P_{k, k+1}(n) \lambda_{j}^{n+k}\left(\zeta^{1 / p}\right) t^{n / p}}{\Gamma(1+n / p)}=0 .
\end{aligned}
$$

Lemma 5. Let $v(t, z) \in \mathcal{O}_{1 / p}\left(D^{2}(r)\right)$ with some $r>0$. Then $v(t, z)$ satisfies the pseudodifferential equation

$$
\left(\partial_{t}^{1 / p}-\lambda_{j}\left(\partial_{z}^{1 / p}\right)\right) v(t, z)=0
$$

if and only if $v(t, z)$ is a solution of

$$
\left(\partial_{z}^{1 / p}-\lambda_{j}^{-1}\left(\partial_{t}^{1 / p}\right)\right) v(t, z)=0
$$

Proof. Since the equations (24) and (25) are symmetric, it is sufficient to show one-way implication. So, let us assume that $v(t, z)$ is a solution of $(24)$. Then

$$
v(t, z)=\sum_{n=0}^{\infty} \frac{\lambda_{j}^{n}\left(\partial_{z}^{1 / p}\right) \varphi(z)}{\Gamma(1+n / p)} t^{n / p} \quad \text { with } \quad \varphi(z):=v(0, z) \in \mathcal{O}_{1 / p}(D(r)) .
$$

Since

$$
\lambda_{j}\left(\partial_{z}^{1 / p}\right) \varphi(z)=\frac{1}{2 p \pi i} \oint_{|w|=\varepsilon}^{p} \varphi(w) \int_{\zeta_{0}}^{\infty(\theta)} \lambda_{j}\left(\zeta^{1 / p}\right) e_{1 / p}(z \zeta) e^{-w \zeta} d \zeta d w,
$$


by (26) we have

$$
\begin{aligned}
v(t, z) & =\frac{1}{2 p \pi i} \oint_{|w|=\varepsilon}^{p} \varphi(w) \int_{\zeta_{0}}^{\infty(\theta)} \sum_{n=0}^{\infty} \frac{\lambda_{j}^{n}\left(\zeta^{1 / p}\right) t^{n / p}}{\Gamma(1+n / p)} e_{1 / p}(z \zeta) e^{-w \zeta} d \zeta d w \\
& =\frac{1}{2 p \pi i} \oint_{|w|=\varepsilon}^{p} \varphi(w) \int_{\zeta_{0}}^{\infty(\theta)} e_{1 / p}\left(\lambda_{j}^{p}\left(\zeta^{1 / p}\right) t\right) e_{1 / p}(z \zeta) e^{-w \zeta} d \zeta d w .
\end{aligned}
$$

Using the substitution $\tau:=\lambda_{j}^{p}\left(\zeta^{1 / p}\right)$ we obtain

$$
\begin{aligned}
v(t, z)= & \frac{1}{2 p \pi i} \oint_{|w|=\varepsilon}^{p} \varphi(w) \times \\
& \times \int_{\tau_{0}}^{\infty(\tilde{\theta})} e_{1 / p}(\tau t) e_{1 / p}\left(z \lambda_{j}^{-p}\left(\tau^{1 / p}\right)\right) e^{-w \lambda_{j}^{-p}\left(\tau^{1 / p}\right)} d \lambda_{j}^{-p}\left(\tau^{1 / p}\right) d w .
\end{aligned}
$$

with $\tilde{\theta}_{j}=\theta_{j}+p \arg \lambda_{j}$. Hence we have

$$
\begin{aligned}
& \partial_{z}^{1 / p} v(t, z)=\frac{1}{2 p \pi i} \oint_{|w|=\varepsilon}^{p} \varphi(w) \times \\
& \times \int_{\tau_{0}}^{\infty(\tilde{\theta})} \lambda_{j}^{-1}\left(\tau^{1 / p}\right) e_{1 / p}(\tau t) e_{1 / p}\left(z \lambda_{j}^{-p}\left(\tau^{1 / p}\right)\right) e^{-w \lambda_{j}^{-p}\left(\tau^{1 / p}\right)} d \lambda_{j}^{-p}\left(\tau^{1 / p}\right) d w \\
& =\lambda_{j}^{-1}\left(\partial_{z}^{1 / p}\right) v(t, z)
\end{aligned}
$$

Lemma 6. Let $v(t, z) \in \mathcal{O}_{1 / p}\left(D^{2}(r)\right)$ with some $r>0$. Then for every $n \in \mathbb{N}$, $v(t, z)$ satisfies the pseudodifferential equation

$$
\left(\partial_{t}^{1 / p}-\lambda_{j}\left(\partial_{z}^{1 / p}\right)\right)^{n} v(t, z)=0
$$

if and only if $v(t, z)$ is a solution of

$$
\left(\partial_{z}^{1 / p}-\lambda_{j}^{-1}\left(\partial_{t}^{1 / p}\right)\right)^{n} v(t, z)=0 .
$$

Proof. The proof is by induction on $n$. By Lemma 5, the statement holds for $n=1$. So, we may assume that assertion holds for $n=k$. We have

$$
\begin{aligned}
& \left(\partial_{t}^{1 / p}-\lambda_{j}\left(\partial_{z}^{1 / p}\right)\right)^{k+1} v(t, z)=0 \Longleftrightarrow \\
& \left(\partial_{t}^{1 / p}-\lambda_{j}\left(\partial_{z}^{1 / p}\right)\right) \tilde{v}(t, z)=0 \text { with } \tilde{v}(t, z):=\left(\partial_{t}^{1 / p}-\lambda_{j}\left(\partial_{z}^{1 / p}\right)\right)^{k} v(t, z) \\
& \text { by } \stackrel{\text { Lemma } 5}{\Longleftrightarrow}\left(\partial_{z}^{1 / p}-\lambda_{j}^{-1}\left(\partial_{t}^{1 / p}\right)\right) \tilde{v}(t, z)=0 \Longleftrightarrow \\
& \left(\partial_{t}^{1 / p}-\lambda_{j}\left(\partial_{z}^{1 / p}\right)\right)^{k} \bar{v}(t, z)=0 \text { with } \bar{v}(t, z):=\left(\partial_{z}^{1 / p}-\lambda_{j}^{-1}\left(\partial_{t}^{1 / p}\right)\right) v(t, z) \\
& \text { by the inductive assumption }\left(\partial_{z}^{1 / p}-\lambda_{j}^{-1}\left(\partial_{t}^{1 / p}\right)\right)^{k} \bar{v}(t, z)=0 \Longleftrightarrow \\
& \left(\partial_{z}^{1 / p}-\lambda_{j}^{-1}\left(\partial_{t}^{1 / p}\right)\right)^{k+1} v(t, z)=0 .
\end{aligned}
$$

Theorem 1. Let $s>1$ and $d \in \mathbb{R}$. Suppose that $v(t, z)$ is a solution of (8) with the initial data (9). Moreover, suppose that $\left\{\lambda_{1}, \ldots, \lambda_{l}\right\}$ is the set of the characteristic 
roots of

$$
P_{m}(\lambda, 1)=\lambda^{m}-\sum_{j=1}^{m} \lambda^{m-j} p_{j}=0 \quad \text { with } \quad p_{j}:=\lim _{\xi \rightarrow \infty} P_{j}(\xi) / \xi^{j} .
$$

Then $v(t, z) \in \mathcal{O}_{1 / p}(S(d, \delta) \times D(r))$ (for some $\delta>0$ and $r>0$ ) with exponential growth of order $s$ as $t \rightarrow \infty$ if and only if for every $j=1, \ldots, l$ and $n=0, \ldots, m-1$, $\varphi_{n}(z) \in \mathcal{O}_{1 / p}\left(S\left(d+p \arg \lambda_{j}, \tilde{\delta}\right)\right)$ (with some $\tilde{\delta}>0$ ) with exponential growth of order $s$ as $z \rightarrow \infty$.

Proof. $(\Longleftarrow)$ Without loss of generality we may assume that the initial data satisfy (10) for $\varphi(z) \in \mathcal{O}_{1 / p}\left(S\left(d+p \arg \lambda_{j}, \tilde{\delta}\right)\right)$ with exponential growth of order $s$ as $z \rightarrow \infty$. By Lemma $3, v(t, z)=\sum_{j=1}^{l} v_{j}(t, z)$, where $v_{j}(t, z) \in \mathcal{O}_{1 / p}(S(d, \delta) \times D(r))$ with exponential growth of order $s$ as $t \rightarrow \infty$. It means that also $v(t, z) \in \mathcal{O}_{1 / p}(S(d, \delta) \times$ $D(r))$ with the same growth condition.

$(\Longrightarrow)$ If $v(t, z) \in \mathcal{O}_{1 / p}(S(d, \delta) \times D(r))$ is a solution of (8) and is of exponential growth of order $s$, then $v(t, z)$ also satisfies the following Cauchy problem in $z$ direction

$$
P\left(\partial_{t}^{1 / p}, \partial_{z}^{1 / p}\right) v(t, z)=0, \quad\left(\partial_{t}^{1 / p}\right)^{n} v(t, 0)=\psi_{n}(t) \quad \text { for } n=0, \ldots, m-1,
$$

where $\psi_{n}(t) \in \mathcal{O}_{1 / p}(S(d, \delta))$ with exponential growth of order $s$ and

$$
\begin{aligned}
P\left(\partial_{t}^{1 / p}, \partial_{z}^{1 / p}\right) & =\left(\partial_{t}^{1 / p}\right)^{m}-\sum_{j=1}^{m}\left(\partial_{t}^{1 / p}\right)^{m-j} P_{j}\left(\partial_{z}^{1 / p}\right) \\
& =c\left(\left(\partial_{z}^{1 / p}\right)^{m}-\sum_{j=1}^{m}\left(\partial_{z}^{1 / p}\right)^{m-j} \tilde{P}_{j}\left(\partial_{t}^{1 / p}\right)\right)
\end{aligned}
$$

with some polynomials $\tilde{P}_{j}(\xi)$ satisfying $\operatorname{deg} \tilde{P}_{j}(\xi) \leq j$ and $\operatorname{deg} \tilde{P}_{m}(\xi)=m$.

As in a previous case, we may put

$\psi_{0}(t):=\psi(t) \in \mathcal{O}_{1 / p}^{s}(S(d, \delta)), \quad \psi_{n}(t):=\sum_{j=1}^{n} \tilde{P}_{j}\left(\partial_{t}^{1 / p}\right) \psi_{n-j}(t) \quad(n=1, \ldots, m-1)$ for some $\psi(t) \in \mathcal{O}_{1 / p}(S(d, \delta))$ with exponential growth of order $s$ as $t \rightarrow \infty$.

Interchanging the roles of coordinates $(t, z)$ and repeating the proof of Lemma 3 we obtain

$$
v(t, z)=\sum_{j=1}^{l} \tilde{v}_{j}(t, z)
$$

where

$$
\begin{aligned}
& \tilde{v}_{j}(t, z)=\frac{1}{2 p \pi i} \oint_{|s|=\varepsilon}^{p} \psi(s) \times \\
& \times \int_{\tau_{0}}^{\infty\left(\tilde{\theta}_{j}\right)} \sum_{k=0}^{m_{j}-1} \tilde{c}_{j k}\left(\tau^{1 / p}\right) r^{k}\left(z, \partial_{z}^{1 / p}\right) e_{1 / p}\left(z \lambda_{j}^{-p}\left(\tau^{1 / p}\right)\right) e_{1 / p}(t \tau) e^{-s \tau} d \tau d s .
\end{aligned}
$$

Moreover, since

$$
d-\arg \left(\lim _{\tau \rightarrow \infty} \lambda_{j}^{-p}\left(\tau^{1 / p}\right) / \tau\right)=d-\arg \lambda_{j}^{-p}=d+p \arg \lambda_{j},
$$

we conclude that $\tilde{v}_{j}(t, z) \in \mathcal{O}_{1 / p}\left(D(\tilde{r}) \times S\left(d+p \arg \lambda_{j}, \tilde{\delta}\right)\right)$ with exponential growth of order $s$ as $z \rightarrow \infty$. 
By Lemmas 4 and $6, \tilde{v}_{j}(t, z)$ satisfies the formula

$$
\left(\partial_{t}^{1 / p}-\lambda_{j}\left(\partial_{z}^{1 / p}\right)\right)^{m_{j}} \tilde{v}_{j}(t, z)=0 \quad \text { for } \quad j=1, \ldots, l .
$$

In a similar way to [4] we define for $j=1, \ldots, l$

$$
P_{j}\left(\partial_{t}^{1 / p}, \partial_{z}^{1 / p}\right):=\left(\partial_{t}^{1 / p}-\lambda_{j}\left(\partial_{z}^{1 / p}\right)\right)^{m_{j}-1} \prod_{k=1, k \neq j}^{l}\left(\partial_{t}^{1 / p}-\lambda_{k}\left(\partial_{z}^{1 / p}\right)\right)^{m_{k}}
$$

and

$$
\bar{v}_{j}(t, z):=P_{j}\left(\partial_{t}^{1 / p}, \partial_{z}^{1 / p}\right) v(t, z) .
$$

Observe that by (27) and (28)

$$
\bar{v}_{j}(t, z)=P_{j}\left(\partial_{t}^{1 / p}, \partial_{z}^{1 / p}\right) \tilde{v}_{j}(t, z)
$$

and, in consequence, $\bar{v}_{j}(t, z) \in \mathcal{O}_{1 / p}\left(D(\tilde{r}) \times S\left(d+p \arg \lambda_{j}, \tilde{\delta}\right)\right)$ with exponential growth of order $s$ as $z \rightarrow \infty$.

Without loss of generality we may assume that

$$
\left(\partial_{t}^{1 / p}\right)^{n} v(0, z)=0 \text { for } n<m-1, \quad\left(\partial_{t}^{1 / p}\right)^{m-1} v(0, z)=\varphi(z) .
$$

Hence also $\bar{v}_{j}(0, z)=\varphi(z)$ and we conclude that $\varphi(z) \in \mathcal{O}_{1 / p}\left(S\left(d+\arg \lambda_{j}, \tilde{\delta}\right)\right)$ with exponential growth of order $s$ for $j=1, \ldots, l$.

\section{Summability of solutions to linear pde With COnstant COEFficients}

In this section we apply Theorem 1 to show the Borel summability properties for linear pde with constant coefficients given by (1).

By Theorem 1 we have

Proposition 6. Let $v(t, z)$ be the solution of the initial value problem (7) and let $s=q /(q-p)$. Then $v(t, z)$ satisfies the properties 1.-3. in Proposition 3 if and only if the Cauchy data $\varphi_{n}(z) \in \mathcal{O}\left(S\left(\left(d+\arg \alpha_{j}+2 k \pi\right) p / q, \tilde{\delta}\right)\right)$ (for some $\tilde{\delta}>0$ ) with exponential growth of order $s$ for $n=0, \ldots, p-1, j=1, \ldots, l, k=0, \ldots, q-1$, where $\left\{\alpha_{1}, \ldots, \alpha_{l}\right\}$ is the set of the characteristic roots satisfying

$$
\alpha^{p}-\sum_{j=1}^{p} \alpha^{p-j} \tilde{p}_{j}=0 \quad \text { with } \quad \tilde{p}_{j}:=\lim _{\zeta \rightarrow \infty} P_{j q / p}(\zeta) / \zeta^{j q / p} .
$$

Proof. $(\Longrightarrow)$ Observe that $v(t, z) \in \mathcal{O}_{1 / p}(S((d+2 k \pi) p / q, \delta) \times D(r))$ (for some $\delta, r>0)$ with exponential growth of order $s$ as $t \rightarrow \infty$. According to Theorem 1 , $\varphi_{n}(z)$ is $1 / p$-analytic on $S\left((d+2 k \pi) p / q+p \arg \lambda_{j}, \tilde{\delta}\right)$ ) (for some $\tilde{\delta}$ ) with exponential growth of order $s$ for $n=0, \ldots, p-1, k=0, \ldots, q-1$ and $j=1, \ldots, \tilde{l}$, where $\left\{\lambda_{1}, \ldots, \lambda_{\tilde{l}}\right\}$ is the set of the roots of the characteristic equation

$$
\lambda^{p q}-\sum_{j=1}^{p} \lambda^{(p-j) q} \tilde{p}_{j}=0 .
$$

Note that $\lambda$ is the root of (30) if and only if $\alpha=\lambda^{q}$ satisfies (29). It means that $\varphi_{n}(z) \in \mathcal{O}_{1 / p}\left(S\left(\left(d+\arg \alpha_{j}+2 \pi k\right) p / q, \tilde{\delta}\right)\right)$ with exponential growth of order s. Moreover, since $\varphi_{n}(z) \in \mathcal{O}\left(D(\tilde{r})\right.$ ) (for some $\tilde{r}>0$ ), we have also $\varphi_{n}(z) \in$ $\mathcal{O}\left(S\left(\left(d+\arg \alpha_{j}+2 \pi k\right) p / q, \tilde{\delta}\right)\right)$ for $n=0, \ldots, p-1, k=0, \ldots, q-1$ and $j=1, \ldots, l$.

$(\Longleftarrow)$ If $\varphi_{n}(z) \in \mathcal{O}_{1 / p}\left(S\left(\left(d+2 \pi k+\arg \alpha_{j}\right) p / q, \tilde{\delta}\right)\right)$ with exponential growth of order $s$ then also $\varphi_{n}(z) \in \mathcal{O}_{1 / p}\left(S\left((d+2 \pi k) p / q+p \arg \lambda_{j}, \tilde{\delta}\right)\right)$ with the same 
growth $(n=0, \ldots, p-1, k=0, \ldots, q-1, j=1, \ldots, \tilde{l})$, where $\left\{\lambda_{1}, \ldots, \lambda_{\tilde{l}}\right\}$ is the set of the characteristic roots of (30). Therefore by Theorem 1 we have $v(t, z) \in$ $\left.\mathcal{O}_{1 / p}(S(d+2 \pi k) p / q, \delta) \times D(r)\right)$ with exponential growth of order $s$ as $t \rightarrow \infty$. Moreover, since $\varphi_{n}(z) \in \mathcal{O}(D(\tilde{r}))$, the Cauchy data in (7) are non-vanishing only for multiplicity of $\left(\partial_{t}^{1 / p}\right)^{q}$-derivative of $v(0, z)$ and the linear operator $P\left(\partial_{t}^{1 / p}, \partial_{z}^{1 / p}\right)$ in (7) depends only on $\left(\partial_{t}^{1 / p}\right)^{q}$ and $\left(\partial_{z}^{1 / p}\right)^{p}$ we obtain the desired conclusion.

Combining Propositions 3, 5 and 6 we have

Theorem 2 (Main theorem). Let $\hat{u}(t, z)$ be a formal power series solution of the initial value problem

$$
\partial_{t}^{p} u(t, z)=\sum_{j=1}^{p} \partial_{t}^{p-j} P_{j q / p}\left(\partial_{z}\right) u(t, z), \quad \partial_{t}^{n} u(0, z)=\varphi_{n}(z) \quad \text { for } n=0, \ldots, p-1,
$$

where $t, z \in \mathbb{C}, p, q \in \mathbb{N}, p<q, \operatorname{deg} P_{j q / p}(\xi) \leq j q / p(j=1, \ldots, p), \operatorname{deg} P_{q}(\xi)=q$ and $\varphi_{n}(z)$ are analytic in a complex neighbourhood of the origin.

Then the formal series $\hat{u}(t, z)$ is $p /(q-p)$-summable in a direction $d$ if and only if the Cauchy data $\varphi_{n}(z)$ are analytically continued to the set $S\left(\left(d+\arg \alpha_{j}+\right.\right.$ $2 \pi k) p / q, \tilde{\delta}$ ) (with some $\tilde{\delta}>0$ ) for $k=0, \ldots, q-1, j=1, \ldots, l$ and this analytic continuation is of exponential growth of order $q /(q-p)$ as $z \rightarrow \infty$, where $\left\{\alpha_{1}, \ldots, \alpha_{l}\right\}$ is the set of the roots satisfying the characteristic equation (29).

\section{Summability of SOlution to FRACTiOnal Linear PDE With CONSTANT COEFFICIENTS}

In this section we will consider the non-Kowalevskian fractional linear pde in two complex variables with constant coefficients. As in the previous section, we apply Theorem 1 to study the summability property for formal solutions of these equations. First, we shall extend the definition of summability to the wider class of formal power series.

Definition 7. Let $\gamma>0$. The Banach space of $\gamma$-analytic functions on $D(r)$, continuous on its closure and equipped with the norm $\|\varphi\|_{r}:=\max _{|z| \leq r}|\varphi(z)|$ is denoted by $\mathbb{E}_{\gamma}(r)$.

The space of formal power series

$$
\hat{u}(t, z)=\sum_{j=0}^{\infty} u_{j}(z) t^{j} \quad \text { with } \quad u_{j}(z) \in \mathbb{E}_{\gamma}(r)
$$

is denoted by $\mathbb{E}_{\gamma}(r)[[t]]$. Moreover, we set $\mathbb{E}_{\gamma}[[t]]:=\bigcup_{r>0} \mathbb{E}_{\gamma}(r)[[t]]$.

Definition 8. Let $\alpha, \gamma>0, k>0$ and $d \in \mathbb{R}$. We say that $\hat{u}(t, z) \in \mathbb{E}_{\gamma}\left[\left[t^{\alpha}\right]\right]$ is $k$-summable in directions $d+2 j \pi / \alpha(j \in \mathbb{Z})$ if and only if the formal power series $\hat{w}(t, z):=\hat{u}\left(t^{1 / \alpha}, z\right)$ is $k / \alpha$-summable with respect to $t$ in a direction $\alpha d$.

Let us suppose that $\hat{u}(t, z)=\sum_{j=0}^{\infty} \frac{u_{j}(z)}{\Gamma(1+\alpha j)} t^{\alpha j}$. Then $\hat{w}(t, z)=\sum_{j=0}^{\infty} \frac{u_{j}(z)}{\Gamma(1+\alpha j)} t^{j}$. Using $k / \alpha$-Borel transform of $\hat{w}(t, z)$ we obtain the series $\sum_{j=0}^{\infty} \frac{u_{j}(z)}{\Gamma(1+\alpha j) \Gamma(1+\alpha j / k)} t^{j}$. By the theory of moment summability, we may replace this one by the following 
$\alpha$-modified $k / \alpha$-Borel transform of $\hat{w}(t, z)$, which is defined by

$$
\mathcal{B}_{\alpha}^{k / \alpha} \hat{w}(t, z):=\sum_{j=0}^{\infty} \frac{u_{j}(z)}{\Gamma(1+j \alpha(1+1 / k))} t^{j}
$$

Observe that this modified transform is connected with the operator $B^{\alpha, \alpha(1+1 / k)}$ by the formula

$$
\mathcal{B}_{\alpha}^{k / \alpha} \hat{w}(t, z)=B^{\alpha, \alpha(1+1 / k)} \hat{u}\left(t^{k /(\alpha(k+1))}, z\right)
$$

It means that we have the following characterisation of $k$-summability

Proposition 7. The formal series $\hat{u}(t, z) \in \mathbb{E}_{\gamma}\left[\left[t^{\alpha}\right]\right]$ is $k$-summable in directions $d+2 j \pi / \alpha(j \in \mathbb{Z})$ if and only if the function $v(t, z):=B^{\alpha, \alpha(1+1 / k)} \hat{u}(t, z)$ satisfies the following conditions:

(1) $z \mapsto v(t, z)$ is $\gamma$-analytic in some complex neighbourhood of origin in $\mathbb{C}$,

(2) $t \mapsto v(t, z)$ is $\alpha(1+1 / k)$-analytic in some complex neighbourhood of origin in $\mathbb{C}$ and is $\alpha(1+1 / k)$-analytically continued to infinity in directions $\frac{d+2 j \pi / \alpha}{1+1 / k}$ $(j \in \mathbb{Z})$ with exponential growth of order $k+1$.

Now, we can prove

Proposition 8. Let $\alpha \in(0,1), m \in \mathbb{N}$ and $\hat{u}(t, z)$ be a formal power series solution of the Cauchy problem

$\left(\partial_{t}^{\alpha}\right)^{m} u(t, z)=\sum_{j=1}^{m}\left(\partial_{t}^{\alpha}\right)^{m-j} P_{j}\left(\partial_{z}\right) u(t, z), \quad\left(\partial_{t}^{\alpha}\right)^{n} u(0, z)=\varphi_{n}(z) \quad n=0, \ldots, m-1$,

where $\operatorname{deg} P_{j}(\xi) \leq j, \operatorname{deg} P_{m}(\xi)=m$ and $\varphi_{n}(z)$ are analytic in some complex neighbourhood of origin. Then $\hat{u}(t, z)$ is $\alpha /(1-\alpha)$-summable in directions $d+2 j \pi / \alpha$ $(j \in \mathbb{Z})$ if and only if $\varphi_{n}(z)$ are analytically continued in directions $\alpha d+\arg \lambda$ with exponential growth of order $1 /(1-\alpha)$ for every $\lambda$ satisfying the characteristic equation

$$
\lambda^{m}-\sum_{j=1}^{m} \lambda^{m-j} p_{j}=0 \quad \text { with } \quad p_{j}:=\lim _{\xi \rightarrow \infty} P_{j}(\xi) / \xi^{j} .
$$

Proof. By Proposition $7, \hat{u}(t, z) \in \mathbb{E}\left[\left[t^{\alpha}\right]\right]$ is $\alpha /(1-\alpha)$-summable in directions $d+$ $2 j \pi / \alpha$ if and only if the function $v(t, z):=B^{\alpha, 1} \hat{u}(t, z) \in \mathcal{O}(S(\alpha d, \delta) \times D(r)$ ) (for some $\delta>0$ and $r>0)$ with exponential growth of order $1 /(1-\alpha)$ as $t \rightarrow \infty$. By Proposition $4, v(t, z)$ is a solution of the Cauchy problem

$$
\partial_{t}^{m} v(t, z)=\sum_{j=1}^{m} \partial_{t}^{m-j} P_{j}\left(\partial_{z}\right) v(t, z), \quad \partial_{t}^{n} v(0, z)=\varphi_{n}(z) \quad n=0, \ldots, m-1 .
$$

By Theorem 1, $v(t, z) \in \mathcal{O}(S(\alpha d, \delta) \times D(r))$ (for some $\delta>0$ and $r>0$ ) with exponential growth of order $1 /(1-\alpha)$ as $t \rightarrow \infty$ if and only if $\varphi_{n}(z) \in \mathcal{O}(S(\alpha d+$ $\left.\arg \lambda_{j}, \tilde{\delta}\right)$ ) (with some $\tilde{\delta}>0$ ) for every $n=0, \ldots, m-1$ and $j=1, \ldots, l$, where $\left\{\lambda_{1}, \ldots, \lambda_{l}\right\}$ is the set of the characteristic roots of $(31)$ and $\varphi_{n}(z)$ is of exponential growth of order $1 /(1-\alpha)$.

Corollary 1. Let $\beta \in(0,2)$. The formal solution $\hat{u}(t, z)$ of the fractional wavediffusion equation

$$
\partial_{t}^{\beta} u(t, z)=\partial_{z}^{2} u(t, z), \quad u(0, z)=\varphi(z) \in \mathcal{O}(D(r)) \quad \text { with some } r>0
$$


is $\beta /(2-\beta)$-summable in directions $d+2 j \pi / \beta(j \in \mathbb{Z})$ if and only if $\varphi(z) \in$ $\mathcal{O}(S(\beta d / 2, \delta) \cup S(\beta d / 2+\pi, \delta))$ (for some $\delta>0$ ) with exponential growth of order $2 /(2-\beta)$.

Proof. It is sufficient to apply Proposition 8 with $\alpha=\beta / 2, m=2, P_{1}(\xi)=0$, $P_{2}(\xi)=\xi^{2}, \varphi_{0}(z)=\varphi(z)$ and $\varphi_{1}(z)=0$. Observe that $\lambda_{1}=1$ and $\lambda_{2}=-1$ are the roots of the characteristic equation.

Moreover, in a similar way to [6] one can generalise this result to the $n$-dimensional diffusion-wave equation $(n>1)$ as follows

Corollary 2. Let $\beta \in(0,2), \Delta_{z}:=\partial_{z_{1}}^{2}+\ldots+\partial_{z_{n}}^{2}$. The formal solution $\hat{u}(t, z)$ of the fractional wave-diffusion equation

$$
\partial_{t}^{\beta} u(t, z)=\Delta_{z} u(t, z), \quad u(0, z)=\varphi(z) \in \mathcal{O}\left(D^{n}(r)\right) \quad \text { with some } r>0
$$

is $\beta /(2-\beta)$-summable in directions $d+2 j \pi / \beta(j \in \mathbb{Z})$ if and only if the function

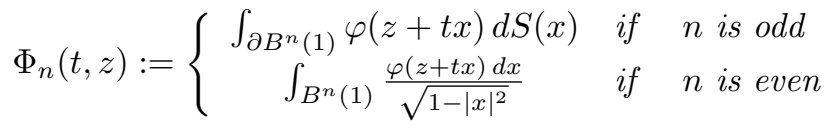

is analytically continued to infinity in some sectors in directions $\beta d / 2$ and $\beta d / 2+\pi$ (with respect to $t$ ) and to some ball with a centre at origin (with respect to $z$ ) and this continuation is of exponential growth of order at most $2 /(2-\beta)$ as $t \rightarrow \infty$.

Proof. By Proposition $7, \hat{u}(t, z) \in \mathbb{E}\left[\left[t^{\beta}\right]\right]$ is $\beta /(2-\beta)$-summable in directions $d+2 j \pi / \beta(j \in \mathbb{Z})$ if and only if the function $v(t, z):=B^{\beta, 2} u(t, z)$ is analytically continued to $\left.(S(\beta d / 2, \delta) \cup S(\beta d / 2+\pi, \delta)) \times D^{n}(\tilde{r})\right)$ (for some $\delta, \tilde{r}>0$ ) with exponential growth of order $2 /(2-\beta)$ as $t \rightarrow \infty$.

On the other hand, $v(t, z)$ is a solution of the wave equation

$$
\partial_{t}^{2} v(t, z)=\Delta_{z} v(t, z), \quad v(0, z)=\varphi(z), \quad v_{t}(0, z)=0 .
$$

The assertion follows by the generalisation of the Kirchhoff and Poisson formula for the solution of the wave equation.

We can also apply the similar methods to the fractional equation with respect to $z$. Namely, we have

Proposition 9. Let $m, p, q \in \mathbb{N}, p>q$ and $\hat{u}(t, z)$ be a formal power series solution of the Cauchy problem

$$
\begin{aligned}
\left(\partial_{t}^{1 / p}\right)^{m} u(t, z) & =\sum_{j=1}^{m}\left(\partial_{t}^{1 / p}\right)^{m-j} P_{j}\left(\partial_{z}^{1 / q}\right) u(t, z) \\
\left(\partial_{t}^{1 / p}\right)^{n} u(0, z) & =\varphi_{n}(z) \text { for } n=0, \ldots, m-1
\end{aligned}
$$

where $\operatorname{deg} P_{j}(\xi) \leq j, \operatorname{deg} P_{m}(\xi)=m$ and $\varphi_{n}(z)$ are $1 / q$-analytic in some complex neighbourhood of origin. Then $\hat{u}(t, z)$ is $q /(p-q)$-summable in a direction $d$ if and only if $\varphi_{n}(z)$ are $1 / q$-analytically continued in directions $q(d / p+\arg \lambda)$ with exponential growth of order $p /(p-q)$ for every $\lambda$ satisfying the characteristic equation (31).

Proof. We repeat the proof of Proposition 8 with $v(t, z):=B^{1 / p, 1 / q} \hat{u}(t, z)$. 
Proposition 10. Let $q \in \mathbb{N}$ and $\alpha \in(0,1 / q), m \in \mathbb{N}$ and $\hat{u}(t, z)$ be a formal power series solution of the Cauchy problem

$$
\begin{aligned}
& \left(\partial_{t}^{\alpha}\right)^{m} u(t, z)=\sum_{j=1}^{m}\left(\partial_{t}^{\alpha}\right)^{m-j} P_{j}\left(\partial_{z}^{1 / q}\right) u(t, z), \\
& \left(\partial_{t}^{\alpha}\right)^{n} u(0, z)=\varphi_{n}(z) \text { for } n=0, \ldots, m-1,
\end{aligned}
$$

where $\operatorname{deg} P_{j}(\xi) \leq j, \operatorname{deg} P_{m}(\xi)=m$ and $\varphi_{n}(z)$ are $1 / q$-analytic in some complex neighbourhood of origin. Then $\hat{u}(t, z)$ is $q \alpha /(1-q \alpha)$-summable in directions $d+$ $2 j \pi / \alpha(j \in \mathbb{Z})$ if and only if $\varphi_{n}(z)$ are $1 / q$-analytically continued in directions $q(\alpha d+\arg \lambda)$ with exponential growth of order $1 /(1-q \alpha)$ for every $\lambda$ satisfying the characteristic equation (31).

Proof. As in a previous case, we repeat the proof of Proposition 8 with $v(t, z):=$ $B^{\alpha, 1 / q} \hat{u}(t, z)$.

\section{REFERENCES}

1. W. Balser, Formal power series and linear systems of meromorphic ordinary differential equations, Springer-Verlag, New York, 2000.

2. _ Summability of formal power-series solutions of partial differential equations with constant coefficients, Journal of Mathematical Sciences 124 (2004), no. 4, 5085-5097.

3. W. Balser and M. Miyake, Summability of formal solutions of certain partial differential equations, Acta Sci. Math. (Szeged) 65 (1999), 543-551.

4. K. Ichinobe, Integral representation for Borel sum of divergent solution to a certain nonKovalevski type equation, Publ. RIMS, Kyoto Univ. 39 (2003), 657-693.

5. D.A. Lutz, M. Miyake, and R. Schäfke, On the Borel summability of divergent solutions of the heat equation, Nagoya Math. J. 154 (1999), 1-29.

6. S. Michalik, Summability of divergent solutions of the $n$-dimensional heat equation, J. Differential Equations 229 (2006), 353-366.

7. _ On the summability of formal solutions to some linear partial differential equations,

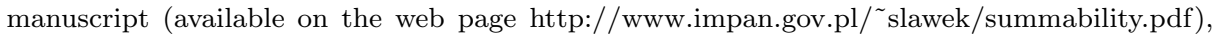
2009.

8. M. Miyake, Borel summability of divergent solutions of the Cauchy problem to non-Kovaleskian equations, Partial Differential Equations and Their Applications, 1999, pp. 225-239.

9. G. Sansone and J. Gerretsen, Lectures on the theory of functions of a complex variable, P. Noordhoff, Groningen, 1960.

Faculty of Mathematics and Natural Sciences, College of Science, Cardinal Stefan Wyszyński University, Wóycickiego 1/3, 01-938 WarszaWa, Poland

Institute of Mathematics Polish Academy of Sciences, P.O. Box 137, Śniadeckich 8 00-950 Warszawa, Poland

E-mail address: s.michalik@uksw.edu.pl

$U R L$ : www.impan.gov.pl/ ${ }^{\text {slawek }}$ 